\title{
A COMPARISON STUDY FOR IMAGE DENOISING
}

\begin{abstract}
Muhammet Fatih ASLAN ${ }^{I^{*}}$, Akif DURDU ${ }^{2}$, Kadir SABANCI ${ }^{3}$
Image denoising is the detection and removal of outliers in a image. A measured analog signal is affected by both the device from which the measurement is performed and the noise from the environment. Various types of noise are available. With the developed noise reduction methods, it is tried to eliminate the existing noise. In this study, Bandelet Transform and Bilateral Filter denoising methods are compared. Both methods have been used to eliminate noise of different types and different rates added to the benchmark and retina images. Bandelet transform is performed for both hard and soft threshold. Peak Signal-to-Noise Ratio, Mean Squared Error, Mean Structural Similarity and Feature Similarity Index are used as a comparison method.
\end{abstract}

Key words: Bandelet transform, Bilateral filter, Image denoising

\section{Introduction}

Today digital images are used in many areas such as intelligent traffic surveillance, medicine, astronomy, satellite television systems, etc. With the increasing need for digital images, denoising has gained importance. Noises cause distortion of spatial resolution in an image and reduce its contrast. Therefore, it negatively affects the edge properties of the image. Processes on noisy images can cause erroneous results. For example, the noise makes difficult to detect tumors and lesions. Thus, denoising must be applied before image analysis.

The images taken with sensors or cameras are usually exposure to noise. No matter how good the cameras are, there is always a need for image enhancement to improve their performance. Noises can result from the device, a data collection process, transmission, compression, environment conditions, etc. $[1,2]$.

The types of noise occurring in the image are varied. Gaussian Noise, Random Noise, Salt and Pepper Noise, Poisson Noise, Speckle Noise, etc. are general noise types [3]. Different types of noise occur in different imaging applications. For example, in the stages of image acquisition or transmission, quantum noise in X-rays and nuclear imaging, speckle noise in ultrasound imaging, and Rician noise in magnetic resonance imaging occur [4]. The structure of the noises is completely different from each other. Therefore, denoising methods can give good results in some filters and bad results in others. In this study, two denoising methods are compared by using three different noise types.

\footnotetext{
${ }^{1}$ Department of Electrical\&Electronics Engineering, Karamanoglu Mehmetbey University, Karaman, Turkey, (mfatihaslan@kmu.edu.tr) Dhttp://orcid.org/0000-0001-7549-0137

${ }^{2}$ Department of Electrical\&Electronics Engineering, Konya Techical University, Konya, Turkey, (adurdu@ktun.edu.tr) http://orcid.org/0000-0002-5611-2322

${ }^{3}$ Department of Electrical\&Electronics Engineering, Karamanoglu Mehmetbey University, Karaman, Turkey, (kadirsabanci@kmu.edu.tr) $@$ http://orcid.org/0000-0003-0238-9606
} 
Although there are many studies [4-11] that have been developed to denoising, most algorithms are not yet at the desired level [12]. Wavelet-based methods have good results in image denoising due to their sparseness and multiple resolution structure. Therefore, many wavelet based algorithms have been developed [13].

Image denoising is the common problem in image processing. Therefore, so much work has been done for image denoising. Zhang, et al. [14] investigated how a high-quality image can be reconstructed from a high-resolution and high-noise astronomical image. For this purpose 2G-bandelet denoising compressed sensing is proposed. As a result, a fast algorithm that preserves more image details and textures is created. Wang and Gao [15] used the second-generation Bandelet Transform (BT) and nonsubsampled contourlet transform as a hybrid. The new denoising method performed better performance than the other two transform. Hazavei and Shahdoosti [16] proposed new multiresolution image denoising method using Bilateral Flter (BF) and complex wavelet thresholding. The advantages of both filters are combined. Experiments on real images showed the effectiveness. He, et al. [17] presented a new retinal image denoising approach that could preserve the details of the retinal vessels while removing image noise. The filter technique used combines the advantages of both BF and matched filter which employs the Gaussian-shape of the cross-section of the vessel. The results showed that this hybrid method was very successful. Finally, in another study by Ceylan and Ozturk [18] a similar performance comparison study was carried out using different denoising methods such as ridgelet, tetrolet, wavelet and curvelet. Deoising methods were evaluated according to the comparison results.

In this study, denoising was applied to the retina and five benchmark images. BT, which is one of the multiple resolution methods, was used with hard and soft thresholding methods which are the most popular threshold methods. Besides this transform, a BF was used to protect the edges and perform a non-linear transformation. The performances of the two methods were compared on three different types of noise

\section{Methodology}

\subsection{Bandelet Transform}

BT proposed by Pennec and Mallat [19] is a transformation adapted to the geometric content of the image. In wavelet-based methods, the same texture values in the image have different directions. To solve this problem, geometric regularity is achieved by using Bandeletization.

BT uses the anisotropic regularity of natural images by creating orthogonal vectors with the direction in which the function has the maximum regularity. BT is a self-adapting multidimensional geometry analysis method that takes advantage of the recognized geometric information of images compared to non-adaptive algorithms such as curvelet and contourlet transformations. The geometric redundancy of an image is removed by bandeletization. In this way, the wavelet transform coefficients are adapted to the image geometry to capture the singularities of the image edges. [20,21].

\subsection{Bilateral Filter}

$\mathrm{BF}$, an alternative to wavelet-based denoising methods, was proposed by Tomasi and Manduchi [22]. Unlike other conventional filters, in BF, both spatial and density information between a point and adjacent points are considered. BF takes the weighted totals of local neighborhood pixels. Each pixel is replaced by the weighted average of its neighbors. The weights are determined to depend on both the 
spatial distance and the intensity distance. Thus, the edges are protected during noise cancellation [23, 24].

\section{Application and Results}

In this study, five benchmark images and 40 fundus images taken from the DRIVE dataset [25] were used. The used benchmark images and some fundus images are shown in Figure 1. First, Random, Gaussian and Rician noises were added to these images respectively (sigma $=5,10,15$ for Random noise; signal-to-ratio (SNR) $=3,5,10$ for Gaussian and Rician noise). Then, these noises were removed by $\mathrm{BT}$ and $\mathrm{BF}$.

A thresholding process was applied to the detail coefficients obtained by BT. In this study, hard and soft thresholding methods were used. After the threshold value was applied, reconstruction was performed. The threshold $\mathrm{T}$ is calculated as follows:

$$
T=\sigma \sqrt{2 \log (M)}
$$

Table 1. Denoising performance results of fundus images

\begin{tabular}{|c|c|c|c|c|c|}
\hline Type of Noise & Noise Ratio & Evaluation Criteria & Bandelet-Hard & Bandelet-Soft & Bilateral Filter \\
\hline \multirow{12}{*}{ Random } & \multirow{4}{*}{ Sigma $=5$} & PSNR & 38,9224 & 34,4843 & 35,7433 \\
\hline & & MSE & 8,3338 & 23,1562 & 17,5101 \\
\hline & & MSSIM & 0,6696 & 0,4798 & 0,3567 \\
\hline & & FSIM & 0,9883 & 0,9248 & 0,9148 \\
\hline & \multirow{4}{*}{ Sigma $=10$} & PSNR & 32,9028 & 28,2764 & 32,5224 \\
\hline & & MSE & 33,3276 & 96,7056 & 36,4667 \\
\hline & & MSSIM & 0,4799 & 0,2752 & 0,3615 \\
\hline & & FSIM & 0,9549 & 0,7915 & 0,9166 \\
\hline & \multirow{4}{*}{ Sigma $=15$} & PSNR & 29,3756 & 24,6809 & 29,8126 \\
\hline & & MSE & 75,0805 & 221,3092 & 67,9410 \\
\hline & & MSSIM & 0,3630 & 0,1839 & 0,3696 \\
\hline & & FSIM & 0,9085 & 0,6764 & 0,9198 \\
\hline \multirow{12}{*}{ Gaussian } & \multirow{4}{*}{ Snr $=3$} & PSNR & 17,3931 & 15,2594 & 19,6052 \\
\hline & & MSE & 1247,3080 & 2038,7836 & 769,5456 \\
\hline & & MSSIM & 0,0400 & 0,0301 & 0,0481 \\
\hline & & FSIM & 0,3919 & 0,3262 & 0,4860 \\
\hline & \multirow{4}{*}{ Snr $=5$} & PSNR & 19,3243 & 17,1597 & 22,5609 \\
\hline & & MSE & 800,4200 & 1316,5242 & 394,6266 \\
\hline & & MSSIM & 0,0532 & 0,0393 & 0,0709 \\
\hline & & FSIM & 0,4526 & 0,3799 & 0,5932 \\
\hline & \multirow{4}{*}{ Snr $=10$} & PSNR & 24,2172 & 21,9646 & 30,9685 \\
\hline & & MSE & 259,5216 & 434,9683 & 57,1614 \\
\hline & & MSSIM & 0,1006 & 0,0775 & 0,1816 \\
\hline & & FSIM & 0,6157 & 0,5327 & 0,8604 \\
\hline \multirow{12}{*}{ Rician } & \multirow{4}{*}{ Snr $=3$} & PSNR & 38,6165 & 26,6154 & 37,6522 \\
\hline & & MSE & 8,9420 & 147,1538 & 11,4536 \\
\hline & & MSSIM & 0,4774 & 0,1643 & 0,3648 \\
\hline & & FSIM & 0,9562 & 0,6947 & 0,9177 \\
\hline & \multirow{4}{*}{ Snr $=5$} & PSNR & 34,2520 & 25,8929 & 37,0590 \\
\hline & & MSE & 24,4282 & 172,4614 & 13,0325 \\
\hline & & MSSIM & 0,3288 & 0,1405 & 0,3723 \\
\hline & & FSIM & 0,9018 & 0,6703 & 0,9233 \\
\hline & \multirow{4}{*}{ Snr $=10$} & PSNR & 28,0683 & 23,9843 & 33,3060 \\
\hline & & MSE & 101,4507 & 263,6581 & 30,4246 \\
\hline & & MSSIM & 0,1743 & 0,1022 & 0,3263 \\
\hline & & FSIM & 0,7629 & 0,6080 & 0,9362 \\
\hline
\end{tabular}

Performance was compared with both methods after the noise was removed. Peak Signal-to-Noise Ratio (PSNR), Mean Squared Error (MSE), Mean Structural Similarity (MSSIM) and Feature Similarity 
Index (FSIM) metrics were used as comparison criteria. The results of Retina and Benchmark images are shown in Table 1 and Table 2.

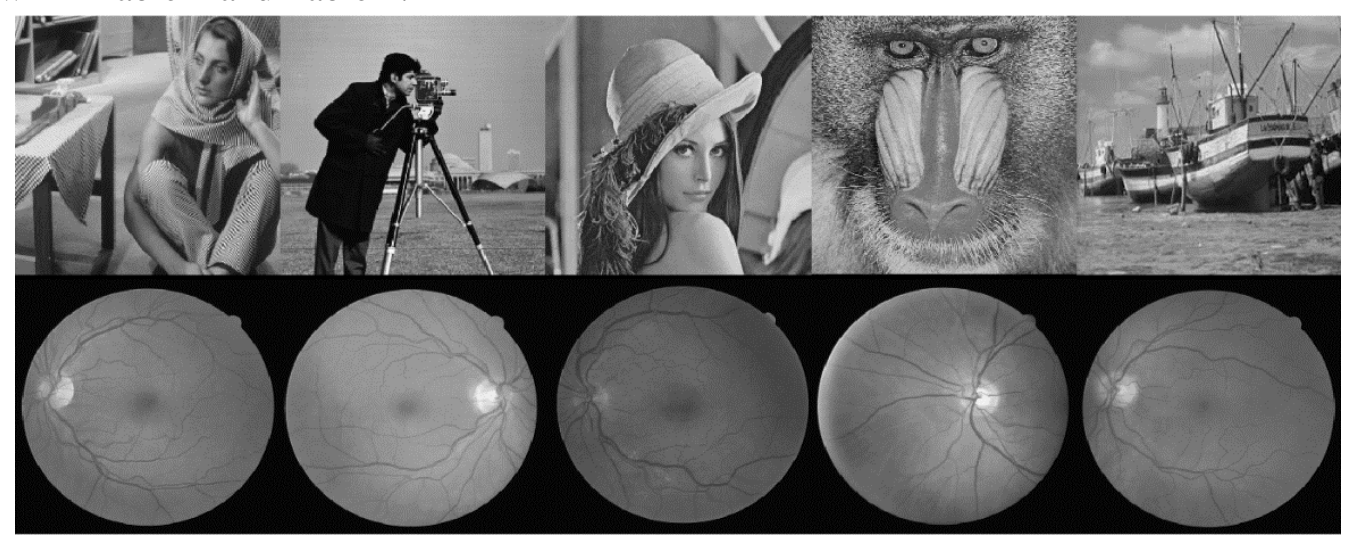

Figure 1. The images used in the application

Table 2. Denoising performance results of benchmark images

\begin{tabular}{|c|c|c|c|c|c|}
\hline Type of Noise & Noise Ratio & Evaluation Criteria & Bandelet-Hard & Bandelet-Soft & Bilateral Filter \\
\hline \multirow{12}{*}{ Random } & \multirow{4}{*}{ Sigma $=5$} & PSNR & 38,9197 & 34,8545 & 31,4374 \\
\hline & & MSE & 8,3390 & 21,2700 & 48,1022 \\
\hline & & MSSIM & 0,9394 & 0,8588 & 0,7156 \\
\hline & & FSIM & 0,9960 & 0,9778 & 0,9073 \\
\hline & \multirow{4}{*}{ Sigma $=10$} & PSNR & 32,9062 & 28,6082 & 29,8290 \\
\hline & & MSE & 33,3014 & 89,6368 & 68,7643 \\
\hline & & MSSIM & 0,8593 & 0,7227 & 0,7119 \\
\hline & & FSIM & 0,9851 & 0,9248 & 0,9067 \\
\hline & \multirow{4}{*}{ Sigma $=15$} & PSNR & 29,3811 & 24,9829 & 28,0706 \\
\hline & & MSE & 74,9854 & 206,5270 & 102,2293 \\
\hline & & MSSIM & 0,7935 & 0,6233 & 0,7105 \\
\hline & & FSIM & 0,9694 & 0,8696 & 0,9076 \\
\hline \multirow{12}{*}{ Gaussian } & \multirow{4}{*}{ Snr=3 } & PSNR & 17,3997 & 14,8921 & 19,4050 \\
\hline & & MSE & 1228,4930 & 2173,6538 & 784,7663 \\
\hline & & MSSIM & 0,2675 & 0,1881 & 0,3044 \\
\hline & & FSIM & 0,6415 & 0,5648 & 0,7055 \\
\hline & \multirow{4}{*}{$\mathrm{Snr}=5$} & PSNR & 19,2674 & 16,7403 & 21,9790 \\
\hline & & MSE & 800,9177 & 1423,5575 & 432,6751 \\
\hline & & MSSIM & 0,3257 & 0,2480 & 0,3767 \\
\hline & & FSIM & 0,6943 & 0,6192 & 0,7744 \\
\hline & \multirow{4}{*}{$\mathrm{Snr}=10$} & PSNR & 24,1006 & 21,5181 & 27,9438 \\
\hline & & MSE & 263,5003 & 475,8568 & 105,6665 \\
\hline & & MSSIM & 0,4874 & 0,4105 & 0,5881 \\
\hline & & FSIM & 0,8121 & 0,7496 & 0,8987 \\
\hline \multirow{12}{*}{ Rician } & \multirow{4}{*}{ Snr=3 } & PSNR & 38,5874 & 25,7080 & 31,9966 \\
\hline & & MSE & 9,0021 & 178,5469 & 42,5126 \\
\hline & & MSSIM & 0,8617 & 0,5664 & 0,7191 \\
\hline & & FSIM & 0,9844 & 0,8435 & 0,9086 \\
\hline & \multirow{4}{*}{ Snr $=5$} & PSNR & 34,1621 & 25,1344 & 31,6675 \\
\hline & & MSE & 24,9396 & 203,2743 & 45,8304 \\
\hline & & MSSIM & 0,7735 & 0,5310 & 0,7194 \\
\hline & & FSIM & 0,9623 & 0,8323 & 0,9091 \\
\hline & \multirow{4}{*}{$\mathrm{Snr}=10$} & PSNR & 28,1726 & 23,4804 & 30,3140 \\
\hline & & MSE & 99,0426 & 295,1275 & 62,1657 \\
\hline & & MSSIM & 0,6165 & 0,4734 & 0,6862 \\
\hline & & FSIM & 0,8964 & 0,7988 & 0,9136 \\
\hline
\end{tabular}




\section{Conclusion}

In this study, an image denoising application was performed comparing the performance of BT and BF. As shown in Table 1 and Table 2, the performance of denoising methods varies at different noise types and different images. In terms of image difference, BF showed better results in fundus images. In the denoising application performed with BT, similar results were obtained in both image types. When examined in terms of noise type, BF was better in both image types in Gaussian noise. BT was generally better in case of random and rician noise. However, as the noise ratio increases, the BF has performed better image denoising.

\section{References}

[1] Buades, A., Coll, B., and Morel, J. M. (2004). On image denoising methods. Technical Note, CMLA (Centre de Mathematiques et de Leurs Applications), 5, pp. 1-40.

[2] Motwani, M. C., Gadiya, M. C., Motwani, R. C., and Harris, F. C. Survey of image denoising techniques." Proc., Proceedings of GSPX, pp. 27-30.

[3] Boyat, A., and Joshi, B. K. Image denoising using wavelet transform and median filtering. Proc., Engineering (NUiCONE), 2013 Nirma University International Conference on, IEEE, pp. 1-6.

[4] Buades, A., Coll, B., and Morel, J.-M. A non-local algorithm for image denoising. Proc., Computer Vision and Pattern Recognition, 2005. CVPR 2005. IEEE Computer Society Conference on, IEEE, pp. 60-65.

[5] Portilla, J., Strela, V., Wainwright, M. J., and Simoncelli, E. P. (2003). Image denoising using scale mixtures of Gaussians in the wavelet domain. IEEE Transactions on Image processing, 12(11), pp. $1338-1351$.

[6] Luisier, F., Blu, T., and Unser, M. (2007). A new SURE approach to image denoising: Interscale orthonormal wavelet thresholding. IEEE Transactions on image processing, 16(3), pp. 593-606.

[7] Elad, M., and Aharon, M. (2006). Image denoising via sparse and redundant representations over learned dictionaries. IEEE Transactions on Image processing, 15(12), pp. 3736-3745.

[8] Dabov, K., Foi, A., Katkovnik, V., and Egiazarian, K. (2007). Image denoising by sparse 3-D transform-domain collaborative filtering. IEEE Transactions on image processing, 16(8), pp. 20802095.

[9] Pu, Y.-F., Zhang, N., Zhang, Y., and Zhou, J.-L. (2016). A texture image denoising approach based on fractional developmental mathematics. Pattern Analysis and Applications, 19(2), pp. 427-445.

[10] Aggarwal, H. K., and Majumdar, A. (2016). Hyperspectral image denoising using spatio-spectral total variation. IEEE Geoscience and Remote Sensing Letters, 13(3), pp. 442-446.

[11] Lahmiri, S., and Boukadoum, M. (2015). A weighted bio-signal denoising approach using empirical mode decomposition. Biomedical Engineering Letters, 5(2), pp. 131-139.

[12] Buades, A., Coll, B., and Morel, J.-M. (2010). Image denoising methods. A new nonlocal principle. SIAM review, 52(1), pp. 113-147.

[13] Kaur, S., and Singh, N. (2014). Image Denoising Techniques: A Review. International Journal of Innovative Research in Computer and Communication Engineering, 2(6).

[14] Zhang, J., Zhang, H., Shi, X., and Geng, S. (2019). High Noise Astronomical Image Denoising via 2G-Bandelet Denoising Compressed Sensing. Optik.

[15] Wang, X., and Gao, J. Image Denoising Method Based on Nonsubsampled Contourlet Transform and Bandelet Transform. Proc., 2009 First International Conference on Information Science and Engineering, pp. 1278-1281.

[16] Hazavei, S. M., and Shahdoosti, H. R. (2017). Using Complex Wavelet Transform and Bilateral Filtering for Image Denoising. arXiv preprint arXiv:1702.01276.

[17] He, Y., Zheng, Y., Zhao, Y., Ren, Y., Lian, J., and Gee, J. (2017). Retinal Image denoising via bilateral filter with a spatial kernel of optimally oriented line spread function. Computational and mathematical methods in medicine, 2017.

[18] Ceylan, M., and Canbilen, A. E. (2017). Performance Comparison of Tetrolet Transform and Wavelet-Based Transforms for Medical Image Denoising. International Journal of Intelligent Systems and Applications in Engineering, 5(4), pp. 222-231. 
[19] Le Pennec, E., and Mallat, S. (2005). Sparse geometric image representations with bandelets. IEEE transactions on image processing, 14(4), pp. 423-438.

[20] Villegas, O. O. V., Domínguez, H. d. J. O., and Sánchez, V. G. C. A comparison of the bandelet, wavelet and contourlet transforms for image denoising. Proc., Artificial Intelligence, 2008. MICAI'08. Seventh Mexican International Conference on, IEEE, pp. 207-212.

[21] Ashraf, R., Bashir, K., Irtaza, A., and Mahmood, M. T. (2015). Content based image retrieval using embedded neural networks with bandletized regions. Entropy, 17(6), pp. 3552-3580.

[22] Tomasi, C., and Manduchi, R. Bilateral filtering for gray and color images. Proc., Computer Vision, 1998. Sixth International Conference on, IEEE, pp. 839-846.

[23] Ahmed, S. S., Messali, Z., Ouahabi, A., Trépout, S., Messaoudi, C., and Marco, S. (2014). Bilateral Filtering and Wavelets based Image Denoising: Application to Electron Microscopy Images with Low Electron Dose. International Journal on Recent Trends in Engineering \& Technology, 11(2), pp. 153-164.

[24] Kumar, B. S. (2013). Image denoising based on non-local means filter and its method noise thresholding. Signal, image and video processing, 7(6), pp. 1211-1227.

[25] Staal, J., Abràmoff, M. D., Niemeijer, M., Viergever, M. A., and Van Ginneken, B. (2004). Ridgebased vessel segmentation in color images of the retina. IEEE transactions on medical imaging, 23(4), pp. 501-509. 